\title{
Two-Dimensional Quantum ring in a Graphene Layer in the presence of a Aharonov-Bohm flux
}

\author{
José Amaro Neto, M. J. Bueno and Claudio Furtadd* \\ Departamento de Física, Universidade Federal da Paraíba, \\ Caixa Postal 5008, 58051-970, João Pessoa, PB, Brazil.
}

\begin{abstract}
In this paper we study the relativistic quantum dynamics of a massless fermion confined in a quantum ring. We use a model of confining potential and introduce the interaction via Dirac oscillator coupling, which provides ring confinement for massless Dirac fermions. The energy levels and corresponding eigenfuctions for this model in graphene layer in the presence of Aharonov-Bohm flux in the centre of the ring and the expression for persistent current in this model are derived. We also investigate the model for quantum ring in graphene layer in the presence of a disclination and a magnetic flux. The energy spectrum and wave function are obtained exactly for this case. We see that the persistent current depends on parameters characterizing the topological defect.

PACS numbers: 73.22.-f,71.55.-i,03.65.Ge
\end{abstract}

Keywords: Topological defects, Dirac oscillator, graphene, quantum ring

* furtado@fisica.ufpb.br 


\section{INTRODUCTION}

With the experimental obtaining of graphene [1], several interesting physical phenomena were observed in this material, such as: anomalous Hall effect [2], Klein paradox [3 5], spin qubits [6], Moiré potential [7], etc. Experiments involving the study of quantum dots in graphene in the presence of magnetic field were performed [8, 9], in which the energy spectrum in this system was observed. Theoretical models of quasiparticles confined in quantum dots [10, 11] and rings [12 14] in graphene have been recently proposed. In a mesoscopic electronic system, it is well known that when one varies the magnetic flux passing through the centre of device of a ring topology, a persistent current arises due to the Aharonov-Bohm effect [15]. This quantum coherence plays a central role in mesoscopic physics. From the theoretical point of view the persistent current has been investigated recently theoretically in Ref. [16-21] and experimentally in [22].

The Dirac oscillator [23] is introduced as the relativistic version of the harmonic oscillator due to the fact that this coupling produces in the non-relativistic limit a harmonic oscillator with a strong spin-orbit coupling. The Dirac oscillator coupling is introduced via the following replacement in Dirac equation: $p \rightarrow p-i M \omega \widehat{\beta} \vec{x}$, where $M$ is a mass of the particle, and $\omega$ is the frequency of the oscillator. The Dirac oscillator has been investigated in several areas of physics: in the study of the hidden supersymmetry by Benitez et al [24]; within the analogy with the Jaynes-Cummings model which was demonstrated by Rozmej and Arvieu [25]; in the presence of topological defects [26]. Additionally, Bermudez et al. [27] have studied the Ramsey interferometric effect in non-relativistic limit of the Dirac oscillator. Recently, applications of Dirac oscillator have been carried out in graphene by Quimbay and Strange [28], in studies of quantum dots by Belouad et al. [29] performed with use of the confining models proposed in Ref. [30, 31]. Thermal properties of the Dirac oscillator have been investigated by Boumali and Hassanabadi [32]. Recently, the Dirac oscillator was observed experimentally in microwave physics [33]. The quantum dot in graphene in the presence of a topological defect was studied by Bueno et al. [34, and spectrum of energy and the current persistent are obtained. In Ref. [34] the confinement potential was coupled in Dirac equation in the way similar to Dirac oscillator [23]. In Ref.[35], the Landau levels in a graphene layer with the presence of disclination was investigated.

In this paper we investigate the quantum ring in a graphene layer. We use the model 
of confining potential proposed in Ref. [36], this model is a relativistic version of the TanInkson model for the confining potential in two-dimensional space [16]. We investigate, in low energy limit, the system described by a massless Dirac equation, where a continuous description near Fermi $K$-points is employed. We use the Dirac oscillator type coupling to confine harmonically the quasiparticles in quantum ring pierced by Aharonov-Bohm flux in a graphene layer. We also study the influence of a disclination in two-dimensional quantum ring of nanometric size in the presence of Aharonov-Bohm quantum flux. From the Landau levels in graphene layer it is possible to obtain the magnitude of magnetic length $\ell_{b}=\sqrt{\hbar / e B} \approx 50 \mathrm{~nm}$. This fact demonstrates the relevance of studying the physical influence of a disclination in this quantum ring, keeping in mind that the average size of one topological defect of this kind is of the order of interatomic distances for the carbon atoms in this structure. In both cases, we obtain the eigenvalues and eigenfunctions of energy and persistent current. In the case of dynamics in the presence of defects, we demonstrate the dependence of these physical quantities on the parameter characterizing the disclination, thus demonstrating the influence of the defect in the dynamics in a quantum ring.

This paper is organized as follows: in section II, we present the confining model and the corresponding coupling in Dirac equation. In Section III, we analyze the quantum dynamics of massless Dirac fermions in a quantum ring in graphene layer, and obtain the exact energy spectrum and eigenfunctions for the model where a thin Aharonov-Bohm flux confined to the center of the ring is introduced, and the persistent current is obtained in this case. In section IV, we obtain the eigenvalues and eingefunctions for quantum dynamics of massless Dirac fermions in a quantum ring in the presence of a disclination, and study the influence of topological defect is investigated and the persistent current, and finally, in section $\mathrm{V}$ we present the concluding remarks.

\section{THE RING CONFINEMENT MODEL}

In this section we present the potential used to confine the quasiparticle in a ring-like topology in graphene layer. Recently in Ref. [36] the authors proposed an extension to relativistic case of Tan-Inkson model [16], which has been constructed to model quantum rings within non-relativistic dynamics of electrons and holes. In this relativistic model of confinement the authors [36] also used two control parameters to obtain a harmonic 
confinement in a two-dimensional ring, and the quantum point limits are obtained when we make one of the parameters to be zero, $a_{1}=0$. In this relativistic model the confining potential is introduced via coupling of the momentum of a quasiparticle in a manner similar to Dirac oscillator [23]. In the non-relativistic limit, the Tan-Inkson potential is obtained. The coupling used in the massless Dirac equation to obtain the harmonic confining potential in ring topology in two-dimensional relativistic system is given by

$$
\mathbf{p} \rightarrow \mathbf{p}+i\left[\frac{\sqrt{2 a_{1}}}{\rho}+\sqrt{2 a_{2}} \rho\right] \gamma^{0} \widehat{e}_{\rho}
$$

where $a_{1}$ and $a_{2}$ are the parameters of the potential. Note that at $a_{1} \longrightarrow 0$ we obtain a quantum dot harmonic confining potential. For the case $a_{2} \longrightarrow 0$ we obtain the relativistic antidot. Now we use this new coupling in a massless Dirac equation to describe a quantum ring structure in graphene in the low-energy limit.

\section{THE QUANTUM RING IN A GRAPHENE LAYER}

The graphene physics can be treated as a laboratory for studies in relativistic quantum mechanics in $(2+1)$-dimensions. In the low energy limit near the Fermi points $\mathbf{K}_{+}$and $\mathbf{K}_{-}$, the dynamics of quasiparticle is described by a relativistic massless fermionic theory. In this theory, the massless Dirac spectrum arises because of the lattice structure of graphene. The hexagonal structure of graphene lattice has a basis composed by two sets of carbon atoms, this symmetry is responsible for the origin of the degree of freedom of isospin. In this energy scale the graphene layer is described by the Dirac equation. Now we introduce the Dirac equation in cylindrical coordinates. We start from the Dirac equation in general coordinate system [37], given by

$$
i \gamma^{\mu} D_{\mu} \Psi+\frac{i}{2} \sum_{k=1}^{3} \gamma^{k}\left[D_{k} \ln \left(\frac{h_{1} h_{2} h_{3}}{h_{k}}\right)\right] \Psi=0
$$

where we defined $v_{f}=\hbar=1$, where $v_{f}$ is the Fermi velocity, and $k=1,2,3$. In this

equation, $D_{\mu}=\frac{1}{h_{\mu}} \frac{\partial}{\partial x^{\mu}}$ corresponds to the derivative in the general coordinate system and the parameters $h_{k}$ are the scale factors. Assuming the cylindrical symmetry, one can write metric in Minkowski space-time as $d s^{2}=-d t^{2}+d \rho^{2}+\rho^{2} d \varphi^{2}$, from which we obtain the scale factors: $h_{0}=1, h_{1}=1, h_{2}=\rho$; and the coordinates: $x^{0}=t, x^{1}=\rho, x^{2}=\varphi$. The matrices 
$\gamma^{\mu}$ are the Dirac matrices defined in Minkowski space-time like:

$$
\gamma^{0}=\widehat{\beta}=\left(\begin{array}{cc}
I & 0 \\
0 & -I
\end{array}\right), \quad \gamma^{i}=\widehat{\beta} \widehat{\alpha}^{i}=\left(\begin{array}{cc}
0 & \sigma^{i} \\
-\sigma^{i} & 0
\end{array}\right)
$$

with $I$ and $\sigma^{i}$ are $2 \times 2$ matrices, being the identity and Pauli matrices respectively. The matrices $\widehat{\alpha}^{i}(i=1,2,3)$ and $\widehat{\beta}$ satisfy the set of properties

$$
\begin{aligned}
& \widehat{\alpha}^{i} \widehat{\alpha}^{j}+\widehat{\alpha}^{j} \widehat{\alpha}^{i}=2 \delta_{i j} I \\
& \widehat{\alpha}^{i} \widehat{\beta}=-\widehat{\beta} \widehat{\alpha}^{i} \\
& \widehat{\alpha}^{i 2}=\widehat{\beta}^{2}=I
\end{aligned}
$$

In graphene, in regions near the Fermi points, the electrons behave like massless fermions, so that (2) is rewritten as

$$
i \gamma^{0} \frac{\partial \Psi}{\partial t}+i \gamma^{1} \frac{\partial \Psi}{\partial \rho}+i \frac{\gamma^{2}}{\rho} \frac{\partial \Psi}{\partial \varphi}+\frac{i \gamma^{1}}{2 \rho} \Psi=0
$$

Using this equation, taking into account the properties of the matrices (3) and 4 , the Dirac equation can be presented as

$$
i \frac{\partial \Psi}{\partial t}=\left[-i \alpha^{1}\left(\frac{\partial}{\partial \rho}+\frac{1}{2 \rho}\right)-\frac{i \alpha^{2}}{\rho}\left(\frac{\partial}{\partial \varphi}\right)\right] \Psi
$$

where we write the Dirac-type Hamiltonian only as $\widehat{H}=\widehat{\alpha} \mathbf{p}$.

Now let us consider a Aharonov-Bohm flux passing through the centre of the ring, and a field described by the vector potential $\mathbf{A}=\frac{\Phi}{2 \pi \rho} \widehat{e}_{\varphi}$. The Aharonov-Bohm flux (magnetic flux) $\Phi$ [38] goes along the $z$-direction. We use the minimal coupling in the momentum $\Pi=\mathbf{p}-|q| \mathbf{A}$ thus changing $\mathbf{p}$ for $\Pi$ so that $\widehat{H}=\widehat{\alpha}(\mathbf{p}-|q| \mathbf{A})$. Thus the Hamiltonian of graphene in the presence of the magnetic field with the coupling (1) is written as

$$
\widehat{H}_{B}=\left[-i \alpha^{1}\left(\frac{\partial}{\partial \rho}+\frac{1}{2 \rho}-\widehat{\beta} \frac{\sqrt{2 a_{1}}}{\rho}-\widehat{\beta} \sqrt{2 a_{2}} \rho\right)-\frac{i \alpha^{2}}{\rho}\left(\frac{\partial}{\partial \varphi}-i \frac{\Phi}{\Phi_{0}}\right)\right]
$$

with $\Phi_{0}=\frac{2 \pi}{|q|}$ is the quantum Aharonov-Bohm magnetic flux. The solution of the Dirac equation is defined by ansatz:

$$
\Psi=e^{-i E t}\left(\begin{array}{l}
\psi \\
\phi
\end{array}\right)
$$


where the spinors $\psi=\psi(\rho, \varphi)$ and $\phi=\phi(\rho, \varphi)$ represent each sublattice of the graphene monolayer. Applying the Hamiltonian (7) in the ansatz (8), we have two coupled equations for $\psi$ and $\phi$ :

$$
E \psi=-i \sigma^{1}\left[\frac{\partial}{\partial \rho}+\frac{1}{2 \rho}+\frac{\sqrt{2 a_{1}}}{\rho}+\sqrt{2 a_{2}} \rho\right] \phi-i \frac{\sigma^{2}}{\rho}\left[\frac{\partial}{\partial \varphi}-i \frac{\Phi}{\Phi_{0}}\right] \phi
$$

and

$$
E \phi=-i \sigma^{1}\left[\frac{\partial}{\partial \rho}+\frac{1}{2 \rho}-\frac{\sqrt{2 a_{1}}}{\rho}-\sqrt{2 a_{2}} \rho\right] \psi-i \frac{\sigma^{2}}{\rho}\left[\frac{\partial}{\partial \varphi}-i \frac{\Phi}{\Phi_{0}}\right] \psi
$$

thus, eliminating $\phi$, and substituting (9) in (10), we obtain the second-order differential equation:

$$
\begin{aligned}
E^{2} \psi & =-\left(1+\frac{1}{\rho^{2}}\right) \frac{\partial^{2} \psi}{\partial \rho^{2}}-\frac{1}{\rho} \frac{\partial \psi}{\partial \rho}+\left(\frac{1}{4}+2 a_{1}-\sqrt{2 a_{1}}+\frac{\Phi^{2}}{\Phi_{0}^{2}}\right) \frac{\psi}{\rho^{2}}+2 a_{2} \rho^{2} \psi+ \\
& +\left(\sqrt{2}+4 \sqrt{a_{1}}\right) \sqrt{a_{2}} \psi \\
& +\frac{2}{\rho^{2}} \frac{\Phi}{\Phi_{0}} \frac{i \partial \psi}{\partial \varphi}+\sigma^{3}\left[\left(\frac{1}{\rho^{2}}-\left(\frac{\sqrt{a_{1}}}{\rho}+\sqrt{a_{2}}\right) 2 \sqrt{2}\right)\left(\frac{\Phi}{\Phi_{0}}+\frac{i \partial}{\partial \varphi}\right)\right] \psi
\end{aligned}
$$

In (11) it is possible to verify that $\psi$ is the eigenfunction of $\sigma^{3}$ whose eigenvalues are $s= \pm 1$, where we write $\sigma^{3} \psi_{s}= \pm \psi_{s}=s \psi_{s}$. We can also see that the operators $\widehat{J}_{z}=-i \frac{\partial}{\partial \varphi}$ and $\widehat{p}_{z}=-i \frac{\partial}{\partial z}$ are compatible with the conserved observables, i.e., they both commute with the Hamiltonian of the right side of equation, so we can take solution of (11) in the form:

$$
\psi=e^{i j \varphi}\left(\begin{array}{c}
R_{+}(\rho) \\
R_{-}(\rho)
\end{array}\right)
$$

where $j=l+\frac{1}{2}$, with $l=0, \pm 1, \pm 2, \ldots$ Substituting 12 in 11 , using $\sigma^{3} \psi_{s}= \pm \psi_{s}=s \psi$, and considering the property of Pauli matrices that $\sigma^{i} \sigma^{i}=I$ and the notation $R_{s}(\rho)=$ $\left(R_{+}(\rho), R_{-}(\rho)\right)$, we obtain the following radial equation:

$$
\left[\frac{d^{2}}{d \rho^{2}}+\frac{1}{\rho} \frac{d}{d \rho}-\frac{\vartheta_{s}^{2}}{\rho^{2}}-2 a_{2} \rho^{2}+\varepsilon_{s}\right] R_{s}(\rho)=0
$$

where we define the following parameters:

$$
\begin{aligned}
\vartheta_{s} & =\varsigma_{s}+s \sqrt{2 a_{1}} \\
\varsigma_{s} & =l+\frac{1}{2}(1-s)-\frac{\Phi}{\Phi_{0}} \\
\varepsilon_{s} & =E^{2}-2 s \sqrt{2 a_{2}} \varsigma_{s}-2 \sqrt{2 a_{2}}-4 \sqrt{a_{1} a_{2}}
\end{aligned}
$$


To solve equation $(13)$, we use the change of the variable $\varrho=\sqrt{2 a_{2}} \rho^{2}$, and obtain

$$
\left[\varrho \frac{d^{2}}{d \varrho^{2}}+\frac{d}{d \varrho}-\frac{\vartheta_{s}{ }^{2}}{4 \varrho}-\frac{\varrho}{4}+\frac{\varepsilon_{s}}{4 \sqrt{2 a_{2}}}\right] R_{s}(\varrho)=0 .
$$

The solution of the equation (16) must be regular at the origin and finite everywhere, i.e., $R_{s} \rightarrow 0$ where $\rho \rightarrow \infty$. The function satisfying these conditions has the form

$$
R_{s}(\varrho)=e^{\frac{-\varrho}{2}} \varrho^{\frac{\left|\vartheta_{s}\right|}{2}} F_{s}(\varrho)
$$

and substituting $(17)$ in $(16)$, we arrive at following equation

$$
\varrho \frac{d^{2} F_{s}}{d \varrho^{2}}+[|\vartheta|+1-\varrho] \frac{d F_{s}}{d \varrho}+\left[\frac{\varepsilon_{s}}{4 \sqrt{2 a_{2}}}-\frac{\left|\vartheta_{s}\right|}{2}-\frac{1}{2}\right] F_{s}(\varrho)=0
$$

which corresponds to confluent hypergeometric equation [39], whose solution is given by

$$
F_{s}={ }_{1} F_{1}(a, b ; z)={ }_{1} F_{1}\left(\frac{\left|\vartheta_{s}\right|}{2}+\frac{1}{2}-\frac{\varepsilon_{s}}{4 \sqrt{2 a_{2}}},\left|\vartheta_{s}\right|+1, \varrho\right) .
$$

Imposing the condition that the solution for hypergeometric series becomes a polynomial of degree $n$, i.e., $\frac{\left|\vartheta_{s}\right|}{2}+\frac{1}{2}-\frac{\varepsilon_{s}}{4 \sqrt{2 a_{2}}}=-n$, and using the definitions of parameters 15 , we obtain the following eigenvalues

$$
\begin{aligned}
E_{n, l}= & \pm\left\{4 \sqrt{2 a_{2}}\left[n+\frac{\left|l+\frac{1}{2}(1-s)-\frac{\Phi}{\Phi_{0}}+s \sqrt{2 a_{1}}\right|}{2}+s \frac{\left(l+\frac{1}{2}(1-s)-\frac{\Phi}{\Phi_{0}}\right)}{2}+1\right]+\right. \\
& \left.+4 \sqrt{a_{1} a_{2}}\right\}^{1 / 2}
\end{aligned}
$$

which is the spectrum of energy for the quasiparticle $(E>0$ is associated electrons and $E<0$ with holes) confined in the two-dimensional quantum ring on a monolayer of graphene. Note that the energy spectrum 20 depends on the control parameters $a_{1}$ and $a_{2}$ and the quantum numbers $n$ and $l$. The energy spectrum depends on the parameter characterizing the quantum ring in the graphene layer and on the Aharonov-Bohm flux. In the limit $a_{1} \longrightarrow 0$ in Eq. (20), we obtain the spectrum of quasiparticle in quantum dot in graphene layer.

The solutions with positive energy $E>0$ describe the dynamics of electrons in the conduction band, while $E<0$ describes the dynamics of holes. Now we obtain the spinors corresponding to positive energy in (20). First of all, we should note that, by using the radial wave functions $(17)$, we can write $(12)$ in the following form

$$
\psi_{s}=e^{i j \varphi} R_{s}(\varrho)=e^{i(l+1 / 2) \varphi} e^{-\sqrt{\frac{a_{2}}{2}} \rho^{2}}\left(2 a_{2}\right)^{\frac{\left|\vartheta_{s}\right|}{4}} \rho^{\left|\vartheta_{s}\right|} \times{ }_{1} F_{1}\left(-n,\left|\vartheta_{s}\right|+1, \sqrt{2 a_{2}} \rho^{2}\right) .
$$


Substituting (21) into (9), we obtain the solution for spinors of $\phi$ given by

$$
\begin{gathered}
\phi=\frac{i}{E} N_{s}\left\{\left[2 \sqrt{2 a_{2}} \rho-\frac{\left|\vartheta_{s}\right|}{\rho}-\frac{1}{2 \rho}+\frac{\sqrt{2 a_{1}}}{\rho}\right] \sigma^{1}{ }_{1} F_{1}\left(-n,\left|\vartheta_{s}\right|+1, \sqrt{2 a_{2}} \rho^{2}\right)\right\} \\
+\frac{i}{E} N_{s}\left\{\frac{2 n \sqrt{2 a_{2}} \rho}{\left|\vartheta_{s}\right|+1} \sigma^{1}{ }_{1} F_{1}\left(-n+1,\left|\vartheta_{s}\right|+2, \sqrt{2 a_{2}} \rho^{2}\right)\right\} \\
+\frac{1}{E} N_{s}\left\{\left[\frac{1}{\rho}\left(l+\frac{1}{2}-\frac{\Phi}{\Phi_{0}}\right)\right] \sigma^{2}{ }_{1} F_{1}\left(-n,\left|\vartheta_{s}\right|+1, \sqrt{2 a_{2}} \rho^{2}\right)\right\},
\end{gathered}
$$

where $N_{s}$ is the normalization constant. In this way, the bispinors can be represented as

$$
\psi(\rho)=\left(\begin{array}{l}
\psi_{+}(\rho) \\
\psi_{-}(\rho)
\end{array}\right)
$$

From equations (8), (22) and (23), it is possible to write the positive energy solutions of the Dirac equation corresponding to the parallel and antiparallel components toward $z$ axis:

$$
\begin{aligned}
& \Psi_{+}=f_{+1} F_{1}\left(-n,\left|\vartheta_{+}\right|+1, \sqrt{2 a_{2}} \rho^{2}\right) \times\left(\begin{array}{c}
1 \\
0 \\
0 \\
\frac{i}{E}\left[2 \sqrt{2 a_{2}} \rho+\frac{\left(\varsigma_{+}-\left|\vartheta_{+}\right|+\sqrt{2 a_{1}}\right)}{\rho}\right]
\end{array}\right) \\
& +\frac{i f_{+}}{E}\left(\frac{2 n \sqrt{2 a_{2}} \rho}{\left|\vartheta_{+}\right|+1}\right){ }_{1} F_{1}\left(-n+1,\left|\vartheta_{+}\right|+2, \sqrt{2 a_{2}} \rho^{2}\right)\left(\begin{array}{l}
0 \\
0 \\
0 \\
1
\end{array}\right)
\end{aligned}
$$

for $s=+1$ e $\psi_{-}(\rho)=0$, by analogy,

$$
\begin{aligned}
& \Psi_{-}=f_{-}{ }_{1} F_{1}\left(-n,\left|\vartheta_{-}\right|+1, \sqrt{2 a_{2}} \rho^{2}\right) \times\left(\begin{array}{c}
0 \\
1 \\
\frac{i}{E}\left[2 \sqrt{2 a_{2}} \rho-\frac{\left(\varsigma_{-}+\left|\vartheta_{-}\right|-\sqrt{2 a_{1}}\right)}{\rho}\right] \\
0
\end{array}\right) \\
& +\frac{i f_{-}}{E}\left(\frac{2 n \sqrt{2 a_{2}} \rho}{\left|\vartheta_{-}\right|+1}\right){ }_{1} F_{1}\left(-n+1,\left|\vartheta_{-}\right|+2, \sqrt{2 a_{2}} \rho^{2}\right)\left(\begin{array}{l}
0 \\
0 \\
1 \\
0
\end{array}\right),
\end{aligned}
$$


for $s=-1$ e $\psi_{+}(\rho)=0$, where the factor $f_{s}$ in equations 24 and $(25)$, is written as

$$
f_{s}=\left(\frac{\sqrt{8 a_{2}} \Gamma\left(\left|\vartheta_{s}\right|+n+1\right)}{\Gamma(n+1)\left[\Gamma\left(\left|\vartheta_{s}\right|+1\right)\right]^{2}}\right)^{1 / 2} \times e^{-i E t} e^{i(l+1 / 2) \varphi} e^{-\sqrt{\frac{a_{2}}{2}} \rho^{2}}\left(2 a_{2}\right)^{\frac{\left|\vartheta_{s}\right|}{4}} \rho^{\left|\vartheta_{s}\right|},
$$

with $\Gamma(n)$ is the Euler gamma function. This solution correspond to the $E>0$ case for massless Dirac equation for a quasiparticle confined in quantum ring in a graphene layer.

\section{A. The Persistent Current for Quantum Ring in Graphene Layer}

In this subsection we use the Byers-Yang relation [40 and the expression of eigenvalues (20) to calculate the persistent current for the quantum ring in graphene. With help of this relation we can obtain the persistent current by differentiating the expression of energy with respect to the magnetic flux

$$
I=-\sum_{n, l} \frac{\partial E_{n, l}}{\partial \Phi}
$$

Substituting equation 20 into the equation (27), we can calculate the persistent currents in the system:

$$
I=\frac{|q|}{2 \pi} \sum_{n, l} \sqrt{2 a_{2}}\left(\frac{ \pm \vartheta_{s}}{\left|\vartheta_{s}\right|}+1\right) \times\left\{4 \sqrt{2 a_{2}}\left[n+\frac{\left|\vartheta_{s}\right|}{2}+s \frac{\varsigma_{s}}{2}+1\right]+4 \sqrt{a_{1} a_{2}}\right\}^{-1 / 2}
$$

the persistent current (28) depends on the control parameters $a_{1}$ and $a_{2}$ and the quantum numbers $n$ and $l$. The current is a periodic function of the Aharonov-Bohm flux $\Phi_{A B}$. Note that we have considered the persistent current for electrons $E>0$. For the case of holes we use similar calculation for the case $E<0$.

\section{QUANTUM RING IN GRAPHENE LAYER WITH DISCLINATION}

In this section we study the quantum ring in a graphene layer in the presence of a topological defect, in this case, a disclination. We use the geometric theory of defects [41] to describe in the continuum limit the graphene layer with a topological defect. In a real crystal lattice, some topological defects can occur. The study of such defects has shown a number of technological applications [42], as well as better understanding of the electronic transport properties, phase transitions and diffusion in condensed matter systems [43]. Among all this, we highlight the topological defects of a structural nature, which have been studied both in 
gravitation and in condensed matter. In both cases, topological defects are associated with symmetry properties of the system. Dislocations are associated to translational symmetry of the lattice, and disclinations are associated with rotational symmetry. From the geometric viewpoint, dislocations are related with torsion of elastic medium and disclinations are associated with curvature of this medium. This topological defect can be obtained by Volterra [44] process. This process can be visualized by "cut and glue" procedure to obtain the topological defect. The studies of curved structures of carbon using massless Dirac equation intended to investigate electronic properties of these structures, have been carried out by several authors. In particular, González, Guinea and Vozmediano [45, 46] investigated a model to describe the fullerene molecules, in these studies the fullerene molecule is described by a spherical model in the presence on a non-Abelian gauge field produced by a magnetic monopole at the centre of the sphere. Recently, the electronic structure of a graphitic nanoparticle was investigated by Osipov, Kochetov and Pudlak [47 49] using a field theory model. Osipov and collaborators investigated disclinations in the conical, spherical and hyperbolic geometries. The local density of states was obtained using the Dirac equation in these geometries. It is well known that the spinor describing the quasiparticles near Fermi points acquires a holonomy being transported around a disclination. This effect can be viewed as a geometrical Aharonov-Bohm effect [50, 51]. This result was generalized to a system with $n$ disclinations, whose resulting configuration is described by an effective defect [52]. The three-dimensional metric describing a layer with topological defect in geometric theory [41] is given by

$$
d s^{2}=d t^{2}-d \rho^{2}-\alpha^{2} \rho^{2} d \varphi^{2}
$$

where $\alpha$ is the intensity of the disclination, it can be written in terms of the angular sector $\theta$, which we removed or inserted in the graphene layer to form the defect, as $\alpha=1 \pm \theta / 2 \pi$. This cut and glue process obeys the symmetry of the honeycomb lattice and the sector $\theta$ is a multiple of $\pi / 3$, so, $\theta= \pm N \pi / 3$ where $N$ is an integer with $0<N<6$. The "cut and glue" procedure to visualize the topological defect is named the Volterra process, when the parameter $\alpha$ within the range $0<\alpha<1$ characterizes a positive disclination, where within the Volterra process we remove an angular sector $-N \pi / 3$ of graphene layer. The $\alpha$ in the range $1<\alpha<\infty$ characterize negative disclinations, i.e., the defects formed through insertion of an angular sector $+N \pi / 3$ within the Volterra process. In a graphene layer, 
disclinations described by the line element (29) correspond to removed sectors or inserted sectors. We can use gauge fields to describe the influence of a disclination in the electronic properties of graphene. First, for the gauge field, its quantum fluxes measure the angular deficit of the cone when a spinor is transported in a parallel manner around the apex in a closed path proving us the variation of the local reference frame along the path. The matrix of parallel transport or holonomy transformation [50 52] are obtained of geometry (29) and responsible by the variation of the local reference frame in the graphene layer and produces a flux that acts in the sublattices $A / B$ of graphene layer with defect and are expressed by following integral

$$
\oint \Gamma_{\mu} d x^{\mu}=\pi(\alpha-1) \sigma^{z}
$$

We have another contribution for the quantum holonomy in the graphene with topological defects and named of the spin flux. This spin flux is responsible for mixing of the Fermi points $K_{ \pm}$[53], and its expression is given by

$$
\oint a_{\mu} d x^{\mu}=-3 \pi(\alpha-1) \tau^{y}
$$

where $\tau^{i}$ are the Pauli matrices. Notice that the holonomy 31 acts in the $K$-space in contrast with the flux $(30)$ which acts in the sublattices $A / B$. In this way, the $\tau^{i}$ Pauli matrices act in the $K$-space and the Pauli matrices $\sigma^{i}$ act in the real space. Note that the expressions $(30)$ and $(31)$ are functions of the parameter $\alpha$ characterizing the presence of a disclination.

In this way, the Dirac equation for massless quasiparticles can be rewritten in the presence of non-Abelian gauge field introduced by the disclination and described in Eq. (31). This equation is given by

$$
\left[i \gamma^{\mu} \frac{\partial}{\partial x^{\mu}}-i \gamma^{\mu} \Gamma_{\mu}-\gamma^{\mu} \frac{a_{\mu}}{\rho}\right] \psi=0
$$

where the $\gamma^{\mu}$ are the Dirac matrices defined in a curved space. The $\Gamma_{\mu}$ term is a spinor connection, being present due to the curved nature of the geometry of the disclinated layer of graphene in a continuous limit. Moreover, we note in 32 the presence of a new term $a_{\mu}$ which represents a non-Abelian gauge field related to the $K$ spin flux. The non-zero nonAbelian gauge field $a_{\mu}$ found in (32) is responsible for the mixing of the Fermi-points $K_{ \pm}$. Basing on the integral (31), one can express the non-zero component for the non-Abelian 
gauge field $a_{\mu}$ in the graphene layer with disclination as

$$
a_{\phi}= \pm \frac{3}{2}(\alpha-1)
$$

where the sign \pm sign is related with the $K_{ \pm}$spin fluxes. In a curved space the $\gamma^{\mu}$ matrices can be expressed in function of triads fields $e_{a}^{\mu}(x)$. In this curved background the Dirac matrices must be defined by $\gamma^{\mu}=e_{a}^{\mu}(x) \gamma^{a}$ and satisfy the anticommutation relation $\left\{\gamma^{a}, \gamma^{b}\right\}=2 \eta^{a b}$, where $\eta^{a b}=\operatorname{diag}(+--)$, is the usual Minkowski metric. From the spinor theory in curved spaces, the $\gamma^{\mu}$ matrices must be defined in terms of field $e_{a}^{\mu}(x)$ and the Dirac matrices in the space-time, that is, $\gamma^{\mu}=e_{a}^{\mu}(x) \gamma^{a}$. Note that the $\gamma^{a}$ matrices satisfy the anticommutation relation $\left\{\gamma^{a}, \gamma^{b}\right\}=2 \eta^{a b}$, where $\eta^{a b}=\operatorname{diag}(+--)$, is the Minkowski metric. Withal, the vielbein fields satisfy that: $g^{\mu \nu}=e_{a}^{\mu} e_{b}^{\nu} \eta^{a b}$. Thus, we use the following form for the fields $e_{\mu}^{a}$ and $e_{a}^{\mu}$ :

$$
e_{\mu}^{a}=\left(\begin{array}{ccc}
1 & 0 & 0 \\
0 & \cos \varphi & -\alpha \rho \operatorname{sen} \varphi \\
0 & \operatorname{sen} \varphi & \alpha \rho \cos \varphi
\end{array}\right), \quad e_{a}^{\mu}=\left(\begin{array}{ccc}
1 & 0 & 0 \\
0 & \cos \varphi & \operatorname{sen} \varphi \\
0 & -\frac{\operatorname{sen} \varphi}{\alpha \rho} & \frac{\cos \varphi}{\alpha \rho}
\end{array}\right)
$$

Now, we use the Maurer-Cartan structure equation for differential forms, $d e^{a}+\omega_{b}^{a} \wedge e^{b}=0$ (condition of zero torsion), where $e^{a}=e_{\mu}^{a} d x^{\mu}$ and $\omega_{b}^{a}=\omega_{\mu b}^{a} \wedge d x^{\mu}$, we obtain $\gamma^{\mu}=e_{a}^{\mu} \gamma^{a}$, and $\gamma^{\mu}$. The metric tensor is defined by (29), and we write it in terms of the triad as follows: $g^{\mu \nu}=e_{a}^{\mu} \eta^{a b} e_{b}^{\nu}$. We can choose a dual 1-forms basis, which describes the curved geometry of layer with the presence of topological defect, by $e^{a}=e_{\mu}^{a} d x^{\mu}$, where $\omega_{1}^{2}=-\omega_{2}^{1}=-(\alpha-1) d \varphi$. In this way, the non- zero component of the spinorial connection is given by

$$
\Gamma_{\varphi}=-\frac{i}{2}(\alpha-1) \sigma^{3}
$$

Now we consider the inclusion of the Aharonov-Bohm flux piercing through the centre of the quantum ring. The vector potential is given by

$$
\mathbf{A}=\frac{\Phi}{2 \pi \rho} \widehat{e}_{\varphi}
$$

and adding the contribution introduced by minimal coupling, one can write the Eq. (32) as

$$
\gamma^{\mu}\left(i \partial_{\mu}-i \Gamma_{\mu}-e A_{\mu}-\frac{a_{\mu}}{\rho}\right) \psi=0
$$


Now, we introduce the confinement potential (1) in Dirac equation using a coupling similar to Dirac oscillator, thus, the equation becomes

$$
\left[i \gamma^{t} \partial_{t}+i \gamma^{\rho}\left(\partial_{\rho}+\left[\frac{\sqrt{2 a_{1}}}{\rho}+\sqrt{2 a_{2}} \rho\right]+\frac{(\alpha-1)}{2 \alpha \rho}\right)+\gamma^{\phi}\left(i \frac{\partial_{\varphi}}{\alpha \rho}+\frac{1}{\rho} \frac{e \Phi}{\Phi_{0}}-\frac{a_{\varphi}}{\rho}\right)\right] \psi=0
$$

where $\Phi_{0}=\frac{|q|}{2 \pi}$. Now, we adopt the same procedure employed by Villalba in Ref. [54]. We apply a transformation $S(\varphi)=\exp \left[-\frac{i \varphi}{2} \sigma^{3}\right]$ to change the representation of Dirac matrices where the matrices $\gamma^{\rho}$ and $\gamma^{\varphi}$ under similarity transformation $S^{-1}(\varphi) \gamma^{\rho} S(\varphi)=\gamma^{1}$ and $S^{-1}(\varphi) \gamma^{\varphi} S(\varphi)=\gamma^{2}$ are changed to $\gamma^{1}$ and $\gamma^{2}$, while we have applied the following transformation in the spin $\psi \longrightarrow S(\varphi) \psi^{\prime}$. This transformation changes the equation (38) to a new form where we eliminated the spinorial connection term of the Dirac equation. Now, we utilize the following ansatz for solution of Dirac equation:

$$
\Psi^{\prime}=e^{-i E t-i \frac{\varphi}{2} \sigma^{3}}\left(\begin{array}{c}
\psi \\
\phi
\end{array}\right)
$$

where $E$ is a constant. From the similarity transformations and the ansatz (39), we obtain the following set of coupled equations:

$$
E \psi=-i \sigma^{1}\left[\frac{\partial}{\partial \rho}+\frac{1}{2 \rho}+\frac{\sqrt{2 a_{1}}}{\rho}+\sqrt{2 a_{2}} \rho\right] \phi-i \sigma^{2}\left[\frac{1}{\alpha \rho} \frac{\partial}{\partial \varphi}+\frac{i a_{\varphi}}{\rho}-\frac{i}{\rho} \frac{\Phi}{\Phi_{0}}\right] \phi
$$

and

$$
E \phi=-i \sigma^{1}\left[\frac{\partial}{\partial \rho}+\frac{1}{2 \rho}-\frac{\sqrt{2 a_{1}}}{\rho}-\sqrt{2 a_{2}} \rho\right] \psi-i \sigma^{2}\left[\frac{1}{\alpha \rho} \frac{\partial}{\partial \varphi}+\frac{i a_{\varphi}}{\rho}-\frac{i}{\rho} \frac{\Phi}{\Phi_{0}}\right] \psi .
$$

By eliminating $\phi$ with use of (41), from (40) we obtain the following equation

$$
\begin{aligned}
E^{2} \psi= & -\frac{\partial^{2} \psi}{\partial \rho^{2}}-\frac{1}{\rho} \frac{\partial \psi}{\partial \rho}- \\
& -\frac{1}{\rho^{2}}\left[\frac{1}{\alpha^{2}} \frac{\partial^{2}}{\partial \varphi^{2}}-\frac{2}{\alpha}\left(\frac{\Phi}{\Phi_{0}}-a_{\varphi}\right) \frac{i \partial}{\partial \varphi}-\frac{1}{4}+\sqrt{2 a_{1}}-2 a_{1}-\left(\frac{\Phi}{\Phi_{0}}-a_{\varphi}\right)^{2}\right] \psi+ \\
& +\sigma^{3}\left[\left(\frac{1}{\rho^{2}}-\frac{2 \sqrt{2 a_{1}}}{\rho^{2}}-2 \sqrt{2 a_{2}}\right)\left(\frac{\Phi}{\Phi_{0}}-a_{\varphi}+\frac{1}{\alpha} \frac{i \partial}{\partial \varphi}\right)\right] \psi+2 a_{2} \rho^{2} \psi+\left(\sqrt{2}+4 \sqrt{a_{1}}\right) \sqrt{a_{2}} \psi .
\end{aligned}
$$

To solve Eq.42, we use the ansatz

$$
\psi=e^{i j \varphi}\left(\begin{array}{c}
R_{+}(\rho) \\
R_{-}(\rho)
\end{array}\right)
$$


where $j=l+\frac{1}{2}$, with $l=0, \pm 1, \pm 2, \ldots$ Substituting $\psi$ in 42 , we obtain a radial equation for $R_{s}$

$$
\left[\frac{d^{2}}{d \rho^{2}}+\frac{1}{\rho} \frac{d}{d \rho}-\frac{\delta_{s}^{2}}{\alpha^{2} \rho^{2}}-2 a_{2} \rho^{2}+\epsilon_{s}\right] R_{s}(\rho)=0
$$

with parameters $\delta_{s}, \kappa_{s}$ and $\epsilon_{s}$ defined as:

$$
\begin{aligned}
\delta_{s} & =\kappa_{s}+\alpha s \sqrt{2 a_{1}} \\
\kappa_{s} & =\left(l+\frac{1}{2}\right)-\alpha \frac{\Phi}{\Phi_{0}}+\alpha a_{\varphi}-\alpha \frac{s}{2}, \\
\epsilon_{s} & =E^{2}-\frac{2 s \sqrt{2 a_{2}}}{\alpha} \kappa_{s}-2 \sqrt{2 a_{2}}-4 \sqrt{a_{1} a_{2}} .
\end{aligned}
$$

To solve the equation 43 , we use the change of variable $\xi=\sqrt{2 a_{2}} \rho^{2}$, and obtain

$$
\left[\xi \frac{d^{2}}{d \xi^{2}}+\frac{d}{d \xi}-\frac{\delta_{s}^{2}}{4 \alpha^{2} \xi}-\frac{\xi}{4}+\frac{\epsilon_{s}}{4 \sqrt{2 a_{2}}}\right] R_{s}(\xi)=0
$$

Doing the asymptotic analysis of Eq. (45), for the limits $R_{s} \rightarrow 0$ and $\rho \rightarrow \infty$, it is possible to present radial equation in the following form:

$$
R_{s}(\xi)=e^{\frac{-\xi}{2}} \xi^{\frac{\left|\delta_{s}\right|}{2 \alpha}} F_{s}(\xi)
$$

Substituting the Eq. (46) in Eq. 45, we obtain the following equation for $F_{s}$ :

$$
\xi \frac{d^{2} F_{s}}{d \xi^{2}}+\left[\frac{|\delta|}{\alpha}+1-\xi\right] \frac{d F_{s}}{d \xi}+\left[\frac{\epsilon_{s}}{4 \sqrt{2 a_{2}}}-\frac{\left|\delta_{s}\right|}{2 \alpha}-\frac{1}{2}\right] F_{s}(\xi)=0,
$$

where Eq. (47) is the hypergeometric equation whose solution is the hypergeometric function:

$$
F_{s}={ }_{1} F_{1}(a, b ; z)={ }_{1} F_{1}\left(\frac{\left|\delta_{s}\right|}{2 \alpha}+\frac{1}{2}-\frac{\epsilon_{s}}{4 \sqrt{2 a_{2}}}, \frac{\left|\delta_{s}\right|}{\alpha}+1, \xi\right) .
$$

The quantization aspect of the radial solution comes from the fact that hypergeometric functions must obey a convergence requirement, which is achieved when the first parameter of Eq. (48) satisfies the condition

$$
\frac{\left|\delta_{s}\right|}{2 \alpha}+\frac{1}{2}-\frac{\epsilon_{s}}{4 \sqrt{2 a_{2}}}=-n
$$

Substituting the parameters ( 44) in Eq. (49), we obtain the energy spectrum for the quasiparticle confined in a quantum ring in graphene layer with disclination given by

$$
\begin{aligned}
E_{n, l} & = \pm\left\{4 \sqrt { 2 a _ { 2 } } \left[n+\frac{\left|\left(l+\frac{1}{2}\right)-\alpha \frac{\Phi}{\Phi_{0}}+\alpha a_{\varphi}-\alpha \frac{s}{2}+\alpha s \sqrt{2 a_{1}}\right|}{2 \alpha}+\right.\right. \\
& \left.\left.+s \frac{\left(\left(l+\frac{1}{2}\right)-\alpha \frac{\Phi}{\Phi_{0}}+\alpha a_{\varphi}-\alpha \frac{s}{2}\right)}{2 \alpha}+1\right]+4 \sqrt{a_{1} a_{2}}\right\}^{1 / 2},
\end{aligned}
$$


where $s= \pm 1$ is related with sublattices $A / B$ and the eigenvalues $E>0$ represents electrons and $E<0$ holes. Note that in the spectrum, there is the dependence on the quantum numbers $n$ and $l$, the parameters $a_{1}$ and $a_{2}$ and the contribution of the non-Abelian gauge field due to the presence of the disclination. Note that, in the limit $\alpha \longrightarrow 1$ we recover the results of the previous section for a quantum ring in graphene layer. If we consider the quantum dot limit $a_{1} \longrightarrow 0$, we obtain the results obtained in Ref. [34] for a quantum dot in the presence of topological defect. In the limit $a_{2} \longrightarrow 0$ we obtain the free quasiparticle case due nature antidot in the presence of disclination in graphene layer.

In the previous section, the solutions with positive energy $E>0$ describe the dynamics of electrons in the conduction band while $E<0$ describes the dynamics of holes in the valence band. For the equation (39), the spinors corresponding to positive energy are given by

$$
\begin{aligned}
\Psi_{+}=f_{+}{ }_{1} F_{1}\left(-n, \frac{\left|\delta_{+}\right|}{\alpha}+1, \sqrt{2 a_{2}} \rho^{2}\right) \times\left(\begin{array}{c}
1 \\
0 \\
0 \\
\frac{i}{E}\left[2 \sqrt{2 a_{2}} \rho+\frac{\left(\kappa_{+}-\left|\delta_{+}\right|+\alpha \sqrt{2 a_{1}}\right)}{\alpha \rho}\right]
\end{array}\right) \\
+\frac{i f_{+}\left(\frac{2 n \sqrt{2 a_{2}} \rho}{E}\right)}{\frac{\left|\delta_{+}\right|}{\alpha}+1}{ }_{1} F_{1}\left(-n+1, \frac{\left|\delta_{+}\right|}{\alpha}+2, \sqrt{2 a_{2}} \rho^{2}\right)\left(\begin{array}{l}
0 \\
0 \\
0 \\
1
\end{array}\right),
\end{aligned}
$$

for $s=+1$ and $\psi_{-}(\rho)=0$, and by analogy again,

$$
\begin{array}{r}
\Psi_{-}=f_{-}{ }_{1} F_{1}\left(-n, \frac{\left|\delta_{-}\right|}{\alpha}+1, \sqrt{2 a_{2}} \rho^{2}\right) \times\left(\begin{array}{c}
0 \\
1 \\
\frac{i}{E}\left[2 \sqrt{2 a_{2}} \rho-\frac{\left(\kappa_{-}+\left|\delta_{-}\right|-\alpha \sqrt{2 a_{1}}\right)}{\alpha \rho}\right] \\
0
\end{array}\right) \\
+\frac{i f_{-}}{E}\left(\frac{2 n \sqrt{2 a_{2}} \rho}{\frac{|\delta-|}{\alpha}+1}\right){ }_{1} F_{1}\left(-n+1, \frac{\left|\delta_{-}\right|}{\alpha}+2, \sqrt{2 a_{2}} \rho^{2}\right)\left(\begin{array}{l}
0 \\
0 \\
1 \\
0
\end{array}\right),
\end{array}
$$


for $s=-1$ and $\psi_{+}(\rho)=0$. The factor $f_{s}=N_{s} e^{-i E t}$ looks like:

$$
f_{s}=\left(\frac{\sqrt{8 a_{2}} \Gamma\left(\frac{\left|\delta_{s}\right|}{\alpha}+n+1\right)}{\Gamma(n+1)\left[\Gamma\left(\frac{\left|\delta_{s}\right|}{\alpha}+1\right)\right]^{2}}\right)^{1 / 2} \times e^{-i E t} e^{i(l+1 / 2) \varphi} e^{-\sqrt{\frac{a_{2}}{2}} \rho^{2}}\left(2 a_{2}\right)^{\frac{\left|\delta_{s}\right|}{4 \alpha}} \rho^{\frac{\left|\delta_{s}\right|}{\alpha}} .
$$

Note that for $\alpha=1$, the non-Abelian gauge field contribution $a_{\varphi}$ goes to zero. In this way, the parameters $\delta_{s}, \kappa_{s}, \epsilon_{s}$ reduce to the parameters $\vartheta_{s}, \varsigma_{s}, \varepsilon_{s}$ respectively. In other words, we recover the case of graphene layer without defect.

\section{A. The persistent current in the presence of disclination}

Now, using the same procedure as in the previous section, we obtain the persistent current for a quantum ring in a graphene layer with a topological defect. We use the expression of eigenvalues (50) and the Byers-Yang relation [40] given by

$$
I=-\sum_{n, l} \frac{\partial E_{n, l}}{\partial \Phi}
$$

In this way, the persistent current for this system is written as:

$$
I=\frac{|q|}{2 \pi} \sum_{n, l} \sqrt{2 a_{2}}\left(\frac{ \pm \delta_{s}}{\left|\delta_{s}\right|}+1\right) \times\left\{4 \sqrt{2 a_{2}}\left[n+\frac{\left|\delta_{s}\right|}{2 \alpha}+s \frac{\kappa_{s}}{2 \alpha}+1\right]+4 \sqrt{a_{1} a_{2}}\right\}^{-1 / 2} .
$$

So, the persistent current is a function of the control parameters $a_{1}$ and $a_{2}$ and the quantum numbers $n$ and $l$. In addition, we also observed the emergence of correction term $a_{\varphi}$. Note that, the persistent current is a function of the parameter characterizing the curved geometry introduced by the topological defect. The expression (55) is a function of the non-Abelian magnetic flux and the Aharonov-Bohm flux presented in the parameter $\kappa_{s}$. The current is an oscillating function of the Aharonov-Bohm flux $\Phi_{0}$, where the oscillation vanishes for the large values of the flux. These fluxes are responsible for the arising of the persistent current. Note that in the limit $a_{1} \longrightarrow 0$ in Eq. (55) we obtain the persistent current for a quantum dot in the presence of disclination [34] given by

$$
I=\frac{|q|}{2 \pi} \sum_{n, l} \sqrt{2 a_{2}}\left(\frac{ \pm \delta_{s}}{\left|\delta_{s}\right|}+1\right) \times\left\{4 \sqrt{2 a_{2}}\left[n+\frac{\left|\delta_{s}\right|}{2 \alpha}+s \frac{\kappa_{s}}{2 \alpha}+1\right]\right\}^{-1 / 2} .
$$

In the limit $\alpha \longrightarrow 1$ in Eq. (56), we recover the persistent current for a quantum ring in a graphene layer obtained in the previous section. Note that we have considered the persistent current for electrons $E>0$. For the case of holes we use similar calculation for the case $E<0$. 


\section{CONCLUSIONS}

In this Contribution we have investigated a quasiparticle in a quantum ring in graphene in two cases with/without the presence of a topological defect. We have described these quasiparticles at low energies by a two-dimensional massless Dirac equation and have used an exactly solvable theoretical model to describe the harmonic confinement in a quantum ring in a graphene layer. We claim that our model can be used as a theoretical description of a quantum ring in suspended graphene, and in a future work we can introduce a contribution of gapped graphene in our model and study the influence of the presence of gap in this model. We discussed the dynamics of Dirac spinors with/without the impacts of topological defects in the presence of a ring harmonic confining potential. The Dirac oscillator coupling is used with a model for the ring with harmonic potential to confine the quasiparticles. We have obtained the spectrum of energy of a quantum ring in the presence of Aharonov-Bohm fluxes and find that the persistent current is a periodic function of Aharonov-Bohm fluxes. The influence of topological defect changes the electronic structure at low energy due to the coupling of the angular momentum with the non-Abelian gauge fields. The energy spectrum depends on the parameter $\alpha$ characterizing the change of the geometry of graphene layer introduced by disclination, and the parameters $a_{1}$ and $a_{2}$ characterizing the confinement potential. We have obtained the expression for the persistent current depending on the parameters characterizing the quantum ring and the topological defect. The current is a periodic function of the Aharonov-Bohm flux and the non-Abelian flux for case in the presence of the topological defect. We conclude that the impact of confinement potential and geometry introduced by topological defects for the electronic properties of graphene is crucial for the realization of the future investigation in quantum computation in these systems [55, 57].

\section{Acknowledgements}

We are grateful to Knut Bakke for interesting discussion. We thank CNPq, CAPES and $\mathrm{CNPq} /$ Universal, for financial support.

[1] K. S. Novoselov, A. K. Geim, S. V. Morozov, D. Jiang, M. I. Katsnelson, I. V. Grigorieva, S. V. Dubonos, and A. A. Firsov, Nature 438, 197 (2005). 
[2] Y. Zhang, Y.-W. Tan, H. L. Stormer, and P. Kim, Nature 438, 201 (2005).

[3] M. I. Katsnelson, K. S. Novoselov, A. K. Geim, Nature Physics 2, 620 (2006).

[4] A. F. Young, P. Kim, Nature Physics, 5, 222 (2009).

[5] N. Stander, B. Huard, and D. Goldhaber-Gordon,Phys. Rev. Lett. 102, 026807 (2009); arXiv: 0806.2319 .

[6] B. Trauzettel, D. V. Bulaev, D. Loss, and G. Burkard, Nature Physics 3, 192, (2007).

[7] D. Subramaniam, F. Libisch, Y. Li et al., Phys. Rev. Lett. 108, 046801, (2012).

[8] L. A. Ponomarenko, F. Schedin, M. I. Katsnelson, R. Yang, E. W. Hill, K. S. Novoselov, and A. K. Geim, Science 320, 356 (2008).

[9] S. Schnez, F. Molitor, C. Stampfer, J. Güttinger, I. Shorubalko, T. Ihn, and K. Ensslin, Appl. Phys. Lett. 94, 012107 (2009); arXiv: 0807.2710.

[10] P. G. Silvestrov and K. B. Efetov, Phys. Rev. Lett. 98, 016802 (2007).

[11] S. Schnez, K. Ensslin, M. Sigrist, and T. Ihn, Phys. Rev. B 78, 195427 (2008).

[12] M D Petrović, F. M. Peeters, A. Chaves. and G. A . Farias, J. Phys.: Condens. Matter 25 $495301(2013)$.

[13] M. Grujić, M. Tadić and F. M. Peeters, Phys Rev. B 87085434 (2013).

[14] D. R. da Costa, A. Chaves, M. Zarenia, J. M. Pereira Jr. G. A. Farias, and F. M. Peeters, Phys Rev. B 89075418 (2014).

[15] A. C. Bleszynski-Jayich et al., Science 326, 272 (2009).

[16] W.-C. Tan and J. C. Inkson, Phys. Rev. B 60, 5626 (1999).

[17] B. Wunsch, T. Stauber, F. Sols, and F. Guinea, Phys. Rev. Lett. 101, 036803 (2008).

[18] M. M. Ma, J. W. Ding and N. Xu, Nanoscale, 1, 387 (2009).

[19] C. Furtado, A. Rosas and S. Azevedo, EPL 7957001 (2001).

[20] L. Dantas and C. Furtado, Phys. Lett. A 3772926 (2013).

[21] L. Dantas, C. Furtado and A. L. Silva Netto, Phys. Lett. A 37911 (2015).

[22] U. Eckern and P. Schwab, Adv. Phys. 44, 387 (1995).

[23] M. Moshinsky and A. Szczepaniak, J. Phys. A: Math. Gen. 22, 1817 (1989).

[24] J. Benítez, R. P. Martínez y Romero, H. N. Núñez-Yépez, and A. L. Salas-Brito, Phys. Rev. Lett. 64, 1643 (1990).

[25] P. Rozmej and R. Arvieu, J. Phys. A32, 5367 (1999).

[26] J. Carvalho, C. Furtado, and F. Moraes, Phys. Rev. A 84, 032109 (2011). 
[27] A. Bermudez, M. A. Martin-Delgado and A. Luis, Phys. Rev. A 77, 033832 (2008).

[28] C. Quimbay, P. Strange, arXiv:1311.2021.

[29] A. Belouad, A. Jellal and Y. Zahidi, Phys. Lett. A 380, 773 (2016).

[30] A. Jellal, A. D. Alhaidari and H. Bahlouli, Phys. Rev. A 80, 012109 (2009).

[31] H. Bahlouli, A. Jellal and Y. Zahidi, Int. J. Geom. Meth. Mod. Phys. 11, 1450036 (2014).

[32] A. Boumali and H. Hassanabadi, Eur. Phys. J. Plus 128, 124 (2013).

[33] J. A. Franco-Villafañe, E. Sadurn, S. Barkhofen, U. Kuhl, F. Mortessagne and T. H. Seligman, Phys. Rev. Lett. 111, 170405 (2013).

[34] M. J. Bueno, J. Lemos de Mello, C. Furtado and A. M. de M. Carvalho, Eur. Phys. J. P 129 201 (2014).

[35] M. J. Bueno, C. Furtado and A. M. de M. Carvalho, Eur. Phys. J. B 8553 (2012).

[36] K. Bakke and C. Furtado, Phys. Lett. A 376, 1269 (2012).

[37] P. Schluter, K. H. Wietschorke, W. Greiner, J. Phys. A: Math. Gen. 161999 (1983).

[38] Y. Aharonov, and D. Bohm, Phys. Rev. 115, 485 (1959).

[39] M. Abramowitz and I. Stegun, Handbook of Mathematical Functions with Formulas, Graphs, and Mathematical Tables, chap. 13, pag. 504, Dover Publications Inc., New York, 1964.

[40] N. Byers, C. N. Yang, Phys. Rev. Lett. 7, 46 (1961).

[41] M. O. Katanaev and I. V. Volovich, Ann. Phys. (N.Y.) 216, 1 (1992).

[42] J. Lahiri, Y. Lin, P. Bozkurt, I. I. Oleynik, M. Batzill, Nature Nanotechnology, 5, 326 (2010).

[43] S. Hyde, S. Andersson, Z. Blum, T. Landg, S. Lidin, B. W. Ninham,

[44] R. A. Puntingan, H. H. Soleng, Class. Quant. Grav. 141129 (1997) .

[45] J. González, F. Guinea and M. A. H. Vozmediano, Phys. Rev. Lett. 69, 172 (1992).

[46] J. González, F. Guinea and M. A. H. Vozmediano, Nucl. Phys. B406, 771 (1993).

[47] V. A. Osipov and E. A. Kochetov. JETP Lett. 73, 562 (2001).

[48] V. A. Osipov and E. A. Kochetov. JETP Lett. 72, 199 (2000).

[49] V. A. Osipov, E. A. Kochetov and M. Pudlak, JETP Lett. 96, 140 (2003).

[50] P. E. Lammert and V. H. Crespi, Phys. Rev. Lett. 85, 5190 (2000).

[51] P. E. Lammert and V. H. Crespi, Phys. Rev. B 69, 035406 (2004).

[52] C. Furtado, F. Moraes and A. M. de M. Carvalho, Phys. Lett. A 372, 5368 (2008).

[53] J. K. Pachos, Contemporary Physics 50, 375 (2009).

[54] V. M. Villalba, Phys. Rev. A 49, 586 (1994). 
[55] K. Bakke, C. Furtado and S. Sergeenkov, Europhys Lett. 87, 30002 (2009).

[56] K. Bakke and C. Furtado, Quantum Inf. Comput. 11, 4444 (2011)

[57] K. Bakke and C. Furtado, Quantum Inf. Process (2012) DOI: 10.1007/s 11128-012-0358-4. 